M.S. Osman*, Hadi Rezazadeh, and Mostafa Eslami

\title{
Traveling wave solutions for (3+1) dimensional conformable fractional Zakharov-Kuznetsov equation with power law nonlinearity
}

https://doi.org/10.1515/nleng-2018-0163

Received October 26, 2018; revised February 3, 2019; accepted February 10, 2019.

\begin{abstract}
In this work, we consider the $(3+1)$ dimensional conformable fractional Zakharov-Kuznetsov equation with power law nonlinearity. Solitary wave solutions, soliton wave solutions, elliptic wave solutions, and periodic (hyperbolic) wave rational solutions are obtained by means of the unified method. The solutions showed that this method provides us with a powerful mathematical tool for solving nonlinear conformable fractional evolution equations in various fields of applied sciences.
\end{abstract}

Keywords: The unified method; Power law nonlinearity; Conformable fractional derivative; Traveling wave solutions

\section{Introduction}

Nonlinear fractional partial differential equations (FPDEs) show a rich variety of nonlinear phenomena, arise in many physical and engineering applications like geophysical fluid mechanics, fluid mechanics, plasma physics, superconductivity, and optics. Therefore, seeking exact solutions of nonlinear FPDEs specifically the nonlinear fractional evolution equations (NLFEEs) plays an important duty in the study of nonlinear physical phenomena [1-9]. It is significant to find new solutions, since either new exact solutions or numerical approximate solutions may provide more information for understanding the physical phenomena. For an overview and recent developments of the

*Corresponding Author: M.S. Osman, Department of Mathematics, Faculty of Science, Cairo University, Giza-Egypt and Department of Mathematics, Duba University College, University of Tabuk, Saudi Arabia, E-mail: mofatzi@sci.cu.edu.eg Hadi Rezazadeh, Faculty of Engineering Technology, Amol University of Special Modern Technologies, Amol, Iran

Mostafa Eslami, Department of Mathematics, Faculty of Mathematical Sciences, University of Mazandaran, Babolsar, Iran local approach to fractional calculus we refer the reader to $[10,11]$ and references therein.

Very recently, Khalil et al. [12] suggested a conformable fractional derivative. The new fractional derivative is very interesting and is getting an increasing of interest [1317]. The conformable fractional derivatives didn't have a physical meaning as the Caputo or Riemann-Liouville derivatives. This situation is a general open problem for fractional calculus. Despite this many physical applications of conformable fractional derivative appear in the literature. Dazhi Zhao and Maokang Luo generalized the conformable fractional derivative and give the physical interpretation of generalized conformable derivative [18]. In addition, with the help of this fractional derivative and some important formulas, one can convert conformable fractional partial differential equations into integer-order differential equations by traveling wave transformation [19]. Later on, many researchers established exact traveling wave solutions of various nonlinear fractional evolution equations via this fractional derivative. For example, Eslami [20] solved nonlinear fractional coupled nonlinear Schrodinger equations by using the Kudryashov method. Kaplan [21] proposed the modified simple equation method and the exponential rational function method to solve the nonlinear conformable time-fractional Boussinesq equation. Korkmaz [22] applied modified Kudryashov method to obtain the exact solutions of the the $(3+1)$ conformable time-fractional Jimbo-Miwa, Zakharov-Kuznetsov and Modified ZakharovKuznetsov equations. Aminikhah et al. [23] used the sub equation method to obtain the exact solutions of the fractional (1+1) and (2+1) regularized long-wave equations which arise in several physical applications, including ion sound waves in plasma. Rezazadeh et al. [24, 25] concerned about the same method for obtaining traveling wave solutions for the conformable fractional generalized Kuramoto-Sivashinsky equation and fractional ZakharovKuznetsov equation with dual-power law nonlinearity. Tariq et al. [26] investigated the new exact solutions of a nonlinear evolution equation that appear in mathematical physics, specifically Cahn-Allen equation by applying 
tanh method. Akbulut et al. [27] obtained exact solutions for (2+1)-dimensional time-fractional Zoomeron equation and the time-fractional third order modified KdV equation via the auxiliary equation method. Ekici et al. [28] proposed the first integral method to study the optical solitons with fractional temporal evolution in presence of Hamiltonian perturbation terms governed by three types of nonlinearity. Cenesiz et al. [29] obtained some exact solutions for time-fractional Burgers equation, modified Burgers equation and Burgers-Korteweg-de Vries equation via the same method. Kurt et al. [30] established some traveling wave solutions for fractional Nizhnik-NovikovVeselov and fractional Klein-Gordon equations via the Exp-Function Method. Tasbozan et al., [31] solved nonlinear fractional Boussinesq and combined KdV-mKdV equations by using Jacobi elliptic function expansion method. Eslami et al. [32, 33] proposed the first integral method and functional variable method to solve the space-time fractional Schrödinger-Hirota equation and the spacetime fractional modified KDV-Zakharov-Kuznetsov equation and fractional Bogoyavlenskii equations, respectively. Hosseini et al. [34] used the ansatz method to obtain the exact solutions of the fractional Klein-Gordon equations with quadratic and cubic nonlinearities. Eslami [35] applied $G^{\prime} / G$-expansion method to obtain the exact solutions of the space-time fractional (2+1)- dimensional dispersive long wave equations. Cenesiz et al. [36] applied the functional variable method to obtain the exact solutions of fractional modified KdV-ZK equation and Maccari system. Kaplan et al. [37] solved (2+1)-dimensional conformable time-fractional Zoomeron equation and the conformable space-time fractional EW equation by using modified simple equation method.

The aim of this paper is to construct exact traveling wave solutions of the $(3+1)$ dimensional conformable fractional Zakharov-Kuznetsov equation with power law nonlinearity (for $\alpha=1$, see [38])

$$
\frac{\partial^{\alpha} u}{\partial t^{\alpha}}+a u^{n} \frac{\partial u}{\partial x}+b \frac{\partial}{\partial x}\left(\frac{\partial^{2} u}{\partial x^{2}}+\frac{\partial^{2} u}{\partial y^{2}}+\frac{\partial^{2} u}{\partial z^{2}}\right)=0,
$$

by using the unified method.

The unified method (UM) [39-45] and its generalized form (GUM) [46-51], the generalized unified method, that introduce a simple algorithm to find the exact solutions and multi-wave solutions respectively in nonlinear evolution equations and nonlinear conformable fractional evolution equations both with constant and variable coefficients.

This paper is organized as follows: In section 2, we recall some basic definitions of the conformable fractional derivative. In section 3 , the key idea of our method is de- scribed. Sections 4 is devoted to the application of the UM for (3+1) dimensional conformable fractional ZakharovKuznetsov equation. Conclusions are outlined in section 5 .

\section{Conformable fractional derivative}

Here, we introduce some basic properties and definitions of the conformable fractional calculus theory which can be found in $[12,13]$.

Definition 2.1 Let $f:(0, \infty) \rightarrow \mathbb{R}$, then, the conformable fractional derivative of $f$ of order $\alpha$ is defined as [12]

$$
{ }_{t} D^{\alpha} f(t)=\lim _{\varepsilon \rightarrow 0} \frac{f\left(t+\varepsilon t^{1-\alpha}\right)-f(t)}{\varepsilon},
$$

for all $t>0, \alpha \in(0,1)$.

If $f$ is $\alpha$-differentiable in some $(0, a), a>0$ and $\lim _{t \rightarrow 0^{+}} D^{\alpha} f(t)$ exists, then by definition

$$
{ }_{t} D^{\alpha}(f)(0)=\lim _{t \rightarrow 0^{+}} D^{\alpha} f(t) .
$$

The new definition satisfies the properties which given in the following theorem.

Theorem 1 Let $\alpha \in(0,1]$, and $f, g$ be $\alpha$-differentiable at a point $t$, then [12]

(i) ${ }_{t} D^{\alpha}(a f+b g)=a{ }_{t} D^{\alpha} f+b{ }_{t} D^{\alpha} g$, for all $a, b \in \mathbb{R}$.

(ii) ${ }_{t} D^{\alpha}\left(t^{\mu}\right)=\mu t^{\mu-\alpha}$, for all $\mu \in \mathbb{R}$.

(iii) ${ }_{t} D^{\alpha} f g=f_{t} D^{\alpha} g+g_{t} D^{\alpha} f$.

(iv) ${ }_{t} D^{\alpha}\left(\frac{f}{\mathrm{~g}}\right)=\frac{\mathrm{g}_{t} D^{\alpha} f-f_{t} D^{\alpha} \mathrm{g}}{\mathrm{g}^{2}}$.

In addition, if $f$ is differentiable, then ${ }_{t} D^{\alpha} f(t)=t^{1-\alpha} \frac{d f}{d t}$.

In [13], T. Abdeljawad established the chain rule for conformable fractional derivatives as in the following theorem.

Theorem 2 Let $f:(0, \infty) \rightarrow \mathbb{R}$ be a function such that $f$ is differentiable and also $\alpha$-differentiable. Let $g$ be a function defined in the range of $f$ and also differentiable; then, one has the following rule

$$
{ }_{t} D^{\alpha}(f \circ g)(t)=t^{1-\alpha} g^{\prime}(t) f^{\prime}(g(t)) .
$$

\section{The description of the UM}

In this section we describe the UM for finding exact solutions of nonlinear conformable fractional evolution equations.

Consider the following nonlinear conformable fractional evolution equation in two variables and a dependent variable $u$ as

$F\left(u, \frac{\partial^{\alpha} u}{\partial t^{\alpha}}, \frac{\partial u}{\partial x}, \frac{\partial u}{\partial y}, \frac{\partial^{2 \alpha} u}{\partial t^{2 \alpha}}, \frac{\partial^{2} u}{\partial x^{2}}, \ldots\right)=0, t \geq 0,0<\alpha \leq 1$, 
where $F$ is a polynomial in its arguments in which the highest order derivatives and nonlinear terms are involved.

To solve Eq. (4), we take the traveling wave transformation

$$
u(x, y, t)=U(\xi), \quad \xi=x+y-\lambda \frac{t^{\alpha}}{\alpha} .
$$

This enables us to use the following changes

$$
\begin{aligned}
& \frac{\partial^{\alpha}}{\partial t^{\alpha}}(.)=-\lambda \frac{d}{d \xi}(.), \frac{\partial}{\partial x}(.)=\frac{d}{d \xi}(.), \frac{\partial}{\partial y}(.)=\frac{d}{d \xi}(.), \\
& \frac{\partial^{2 \alpha}}{\partial t^{2 \alpha}}(.)=\lambda^{2} \frac{d^{2}}{d \xi^{2}}(.), \ldots
\end{aligned}
$$

Substituting Eq. (5) into Eq. (4) yields a nonlinear ordinary differential equation as following

$$
G\left(U, U^{\prime}, U^{\prime \prime}, \ldots\right)=0,
$$

where $U=U(\xi), U^{\prime}=\frac{d U}{d \xi}, U^{\prime \prime}=\frac{d^{2} U}{d \xi^{2}}, \ldots$.

The obtained solutions of Eq. (6) by using UM are classified to be the polynomial function solutions or the rational function solutions.

\subsection{The polynomial function solution}

To get the polynomial function solutions of Eq. (6), UM suggests that

$$
\begin{aligned}
& U=U(z)=\sum_{i=0}^{n} p_{i} \phi^{i}(z), \\
& \left(\phi^{\prime}(z)\right)^{p}=\sum_{i=0}^{p k} c_{i} \phi^{i}(z), z=\alpha_{0} t+\sum_{s=1}^{q} \alpha_{s} x_{s}, \\
& j=1,2, \ldots, m, p=1,2,
\end{aligned}
$$

where $p_{i}, c_{i}, \alpha_{0}$, and $\alpha_{s}$ are arbitrary constants to be determined later. It is worth to be noticing that, $n$ and $k$ are determined from the balance equation by the criteria given in [39-45]. Also, a second condition (the consistency condition), which asserts that the arbitrary functions in Eq. (7) could be consistently determined, is used.

When $p=1$, (7) solves to elementary solutions (explicit or implicit) while when $p=2$, it solves to elliptic solutions.

\subsection{The rational function solution}

To get the rational function solutions of Eq. (6), UM suggests that

$$
\begin{aligned}
& U=U(z)=\sum_{\substack{i=0 \\
p k}}^{n} p_{i} \phi^{i}(z) / \sum_{i=0}^{r} q_{i} \phi^{i}(z), \quad n \geq r \\
& \left(\phi^{\prime}(z)\right)^{p}=\sum_{i=0}^{p k} c_{i} \phi^{i}(z), z=\alpha_{0} t+\sum_{s=1}^{q} \alpha_{s} \chi_{s}, \\
& j=1,2, \ldots, m, \quad p=1,2,
\end{aligned}
$$

where $p_{i}, q_{i}, c_{i}, \alpha_{0}$, and $\alpha_{s}$ are arbitrary constants to be determined later. It is worth to be noticing that, $n, r$ and $k$ are determined from the balance equation by the criteria given in [39-45]. Also, a second condition (the consistency condition), which asserts that the arbitrary functions in Eq. (8) could be consistently determined, is used.

When $p=1$, (8) solves to elementary solutions (explicit or implicit) while when $p=2$, it solves to elliptic solutions.

\subsection{Steps of computation}

When substituting from Eq. (7) (or Eq. (8)) into Eq. (6), we get the principle equations and the following steps are done.

1-Solving the principle equations.

2-Solving the auxiliary equations.

3-Finding the exact solution.

4-We check that the obtained solution satisfies Eq. (4).

\section{The $(3+1)$ dimensional conformable fractional Zakharov-Kuznetsov equation with power law nonlinearity}

In Eq.(1), $a$ and $b$ are real valued constants. The first term is the evolution term, while the coefficients of $a$ and $b$ respectively, are the nonlinearity and dispersion. Also the parameter $n$ is the power law nonlinearity parameter. Solitons are the result of a delicate balance between dispersion and nonlinearity. Eq.(1) typically appears in the study of plasma physics. B. T. Matebese et al. [52] solved the (3+1) dimensional Zakharov-Kuzetsov equation by $G^{\prime} / G$-expansion method, extended tanh-function method and ansatz metod. Furthermore, Aminikhah et al. [53] proposed the functional variable method to solve this equation when $\alpha=1$. The special case where $n=1$ and $\alpha=1$ gives the (3+1) dimensional Zakharov-Kuzetsov equation [54]. Let

$$
U(\xi)=u(x, y, z, t), \quad \xi=x+y+z-\lambda \frac{t^{\alpha}}{\alpha},
$$

with puting the relation (9) and its derivatives in to the Eq.(1)

$$
\begin{gathered}
-\lambda U_{\xi}+a U^{n} U_{\xi}+b\left(U_{\xi \xi}+U_{\xi \xi}+U_{\xi \xi}\right)_{\xi}=0 . \\
-\lambda U+\frac{a}{n+1} U^{n+1}+3 b U_{\xi \xi}=0 .
\end{gathered}
$$


By using the transformation $U=V^{\frac{1}{n}}$, Eq.(11) can be written as

$$
\begin{aligned}
& 3 b n(n+1) V V_{\xi \xi}+3 b\left(n^{2}-1\right) V_{\xi}^{2}-n^{2}(1+n) \lambda V^{2}+a n^{2} V^{3}, \\
& n \neq 0, n \neq \pm 1 .
\end{aligned}
$$

\subsection{The polynomial solutions}

To find the polynomial solutions of the fractional $(3+1)$ Zakharov-Kuznetsov equation with power law nonlinearity, we assume that

$$
\begin{aligned}
& V(\xi)=\sum_{i=0}^{n} p_{i} \phi^{i}(\xi), \\
& \left(\phi^{\prime}(\xi)\right)^{p}=\sum_{i=0}^{p k} b_{i} \phi^{i}(\xi), \quad p=1,2,
\end{aligned}
$$

where $p_{i}$ and $b_{i}$ are arbitrary constants. By considering the homogeneous balance between $V V_{\xi \xi}$ and $V^{3}$ in Eq.(12), we get $n=2(k-1), k=2,3, \ldots \ldots$

Here, we confine ourselves to find these solutions when $k=2$ and $p=1$ or $p=2$. So, we suppose that the polynomial solution of the ODE (12) has the form

$$
\begin{gathered}
V(\xi)=p_{0}+p_{1} \phi(\xi)+p_{2} \phi^{2}(\xi) \\
\left(\phi^{\prime}(\xi)\right)^{p}=\sum_{i=0}^{2 p} b_{i} \phi^{i}(\xi), \quad p=1,2 .
\end{gathered}
$$

\subsubsection{The solitary wave solution}

To obtain these solutions, we put $p=1$ in the auxiliary equation given by (14). From Eq.(14) when $p=1$, we have

$$
\begin{aligned}
V(\xi) & =p_{0}+p_{1} \phi(\xi)+p_{2} \phi^{2}(\xi), \\
\phi^{\prime}(\xi) & =b_{0}+b_{1} \phi(\xi)+b_{2} \phi^{2}(\xi) .
\end{aligned}
$$

When we use Eq.(15) into Eq.(12) and equating the coefficients of $\phi(\xi)$ to zero we get a system of algebraic equations. By solving this algebraic system of equations with the help of MATHEMATICA or MAPLE, it yields the following:

$$
\begin{aligned}
& p_{0}=-\frac{3 b\left(2+3 n+n^{2}\right)\left(b_{1}^{2}-R^{2}\right)}{2 a n^{2}}, \\
& p_{1}=-\frac{6 b b_{1} b_{2}\left(2+3 n+n^{2}\right)}{a n^{2}}, \\
& p_{2}=-\frac{6 b b_{2}^{2}\left(2+3 n+n^{2}\right)}{a n^{2}}, \lambda=\frac{3 b R^{2}}{n^{2}},
\end{aligned}
$$

By solving the auxiliary equation $\phi^{\prime}(\xi)=b_{0}+b_{1} \phi(\xi)+$ $b_{2} \phi^{2}(\xi)$ and substituting together with (16) into Eq.(12), we get the solution of Eq.(1) namely

$$
u_{1}(x, y, z, t)=\left(\frac{3 b\left(2+3 n+n^{2}\right) R^{2} \operatorname{sech}^{2}\left(\frac{1}{2} R \xi\right)}{2 a n^{2}}\right)^{\frac{1}{n}},
$$

where $\xi=x+y+z-\left(\frac{3 b R^{2}}{n^{2} \alpha}\right) t^{\alpha}$ and $0<\alpha \leq 1$.

Fig. 1 depicts the 3D and 2D charts of the solution given by $u_{1}(x, y, z, t)$ with the parameters $a=0.1, b=0.2, n=$ 2 , and $R=1$.

\subsubsection{The soliton wave solution}

Here, we put $p=2$ in the auxiliary equation given by (14). From Eq.(14) when $p=2$, we have

$$
\begin{gathered}
V(\xi)=p_{0}+p_{1} \phi(\xi)+p_{2} \phi^{2}(\xi), \\
\phi^{\prime}(\xi)=\phi(\xi) \sqrt{b_{0}+b_{1} \phi(\xi)+b_{2} \phi^{2}(\xi) .}
\end{gathered}
$$

By substituting from (18) into Eq.(12) and by a similar way as we did in the last case, we get

$$
\begin{aligned}
& p_{0}=-\frac{9 b b_{1}^{2}}{8 a b_{2}}, \quad p_{1}=-\frac{9 b b_{1}}{a}, \quad p_{2}=-\frac{18 b b_{2}}{a}, \\
& \lambda=-\frac{3 b b_{1}^{2}}{8 b_{2}}, \quad b_{0}=\frac{b_{1}^{2}}{4 b_{2}}, \quad n=2 .
\end{aligned}
$$

By solving the auxiliary equation $\phi^{\prime}(\xi)=$ $\phi(\xi) \sqrt{b_{0}+b_{1} \phi(\xi)+b_{2} \phi^{2}(\xi)}$ and substituting together with (19) into Eq.(18), we get the solution of eq.(1) namely

$$
u_{2}(x, y, z, t)=\frac{3 \sqrt{-b}\left(b_{1}+2 b_{1} b_{2} e^{\frac{b_{1} \xi}{2 \sqrt{b_{2}}}}\right)}{2 \sqrt{2 a b_{2}}\left(1-2 b_{2} e^{\frac{b_{1} \xi}{2 \sqrt{b_{2}}}}\right)}, \quad b<0,
$$

where $\xi=x+y+z+\left(\frac{3 b b_{1}^{2}}{8 b_{2} \alpha}\right) t^{\alpha}$ and $0<\alpha \leq 1$.

Fig. 2 depicts the 3D and 2D charts of the solution given by $u_{2}(x, y, z, t)$ with the parameters $a=0.1, b=$ $-0.2, b_{1}=1$, and $b_{2}=1$.

\subsubsection{The elliptic wave solution}

In this section we find the complex elliptic wave solution. To this end, we put $p=2$ in the auxiliary equation given by (14). From Eq.(14) when $p=2$, we have

$$
\begin{gathered}
V(\xi)=p_{0}+p_{1} \phi(\xi)+p_{2} \phi^{2}(\xi), \\
\phi^{\prime}(\xi)=\sqrt{b_{0}+b_{2} \phi^{2}(\xi)+b_{4} \phi^{4}(\xi)} .
\end{gathered}
$$

where $R=\sqrt{b_{1}^{2}-4 b_{0} b_{2}}$. 

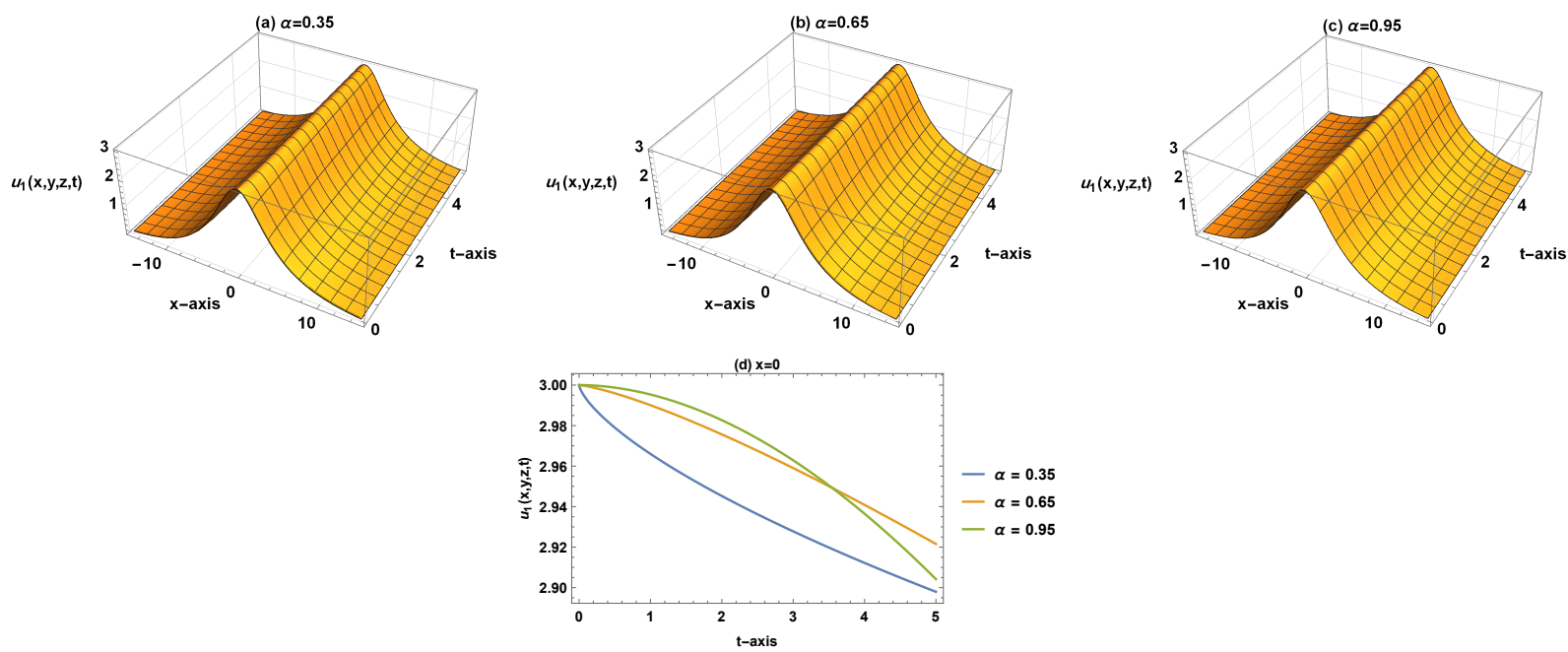

Fig. 1: (a)-(d) The wave solution $u_{1}(x, y, z, t)$ given by (17) in 3D- and 2D-plots when $y=z=0$.
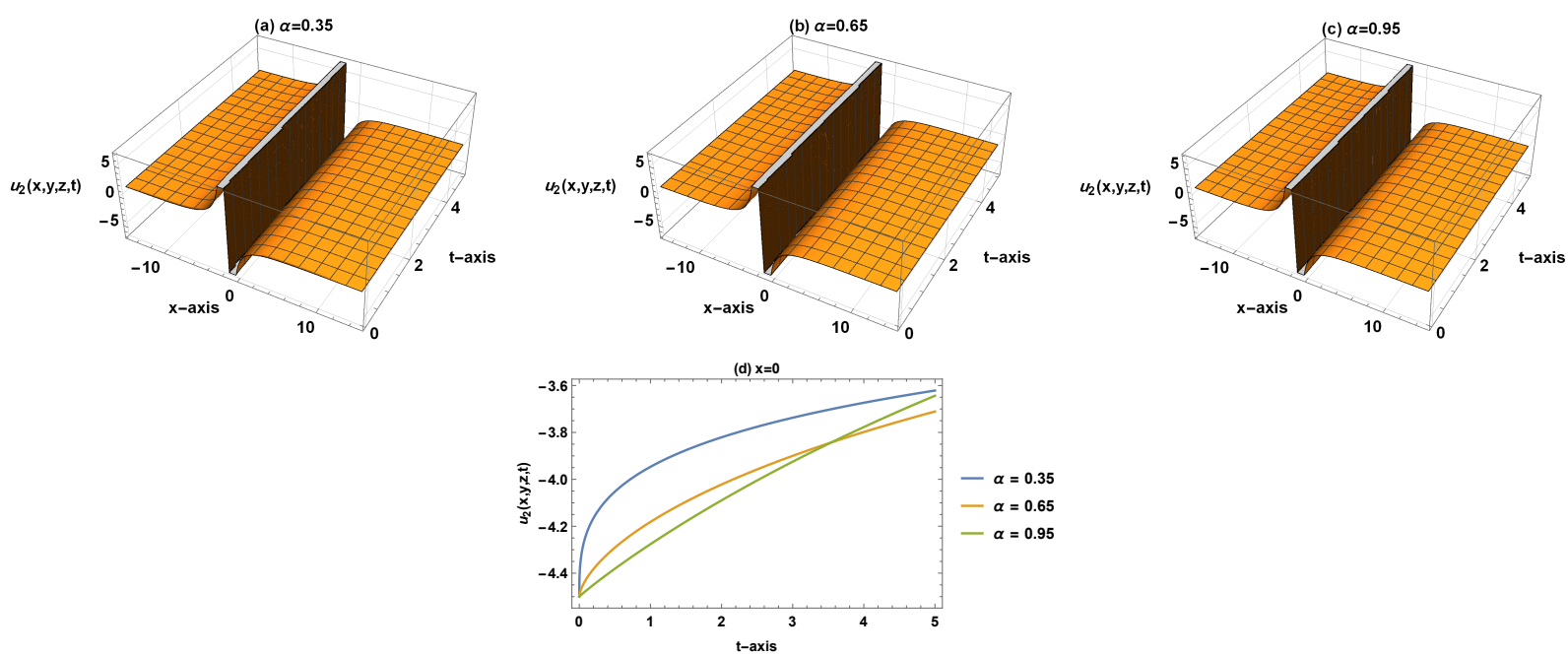

Fig. 2: (a)-(d) The wave solution $u_{2}(x, y, z, t)$ given by (20) in 3D- and 2D-plots when $y=z=0$.

By substituting from (21) into Eq. (12) and by a similar way as we did in the last two cases, we get

$$
\begin{aligned}
& p_{0}=\frac{9 b\left(-b_{2}+\sqrt{b_{2}^{2}-4 b_{0} b_{4}}\right)}{a}, \quad p_{1}=0, \quad p_{2}=-\frac{18 b b_{4}}{a}, \\
& \lambda=\frac{3}{2}\left(-b_{2}+3 \sqrt{b_{2}^{2}-4 b_{0} b_{4}}\right), \quad n=2,
\end{aligned}
$$

where, $b_{i}, i=0,2,4$ are arbitrary constants. For particular values of $b_{i}$ we get different solutions in Jacobi elliptic functions. According to the classification in [55], namely

$$
b_{4}=-\frac{1}{4}, b_{2}=\frac{1+k^{2}}{2}, b_{0}=-\frac{\left(1-k^{2}\right)^{2}}{2}, 0<k<1,
$$

the auxiliary function takes the form $\phi(\xi)=k \mathrm{cn}(\xi, k)+$ $\operatorname{dn}(\xi, k)$ and the solution of Eq. (1) will be in the form

$$
\begin{aligned}
& u_{3}(x, y, z, t)=\left(\frac { 9 b } { 2 a } \left(-1-k^{2}+\sqrt{-k^{4}+6 k^{2}-1}\right.\right. \\
& \left.\left.+(k \operatorname{cn}(\xi, k)+\operatorname{dn}(\xi, k))^{2}\right)\right)^{\frac{1}{2}},
\end{aligned}
$$

where $\xi=x+y+z-\frac{3}{4} b\left(-1-k^{2}+3 \sqrt{-1+6 k^{2}-k^{4}}\right)\left(\frac{t^{\alpha}}{\alpha}\right)$ and $0<\alpha \leq 1$. We mention that $0<k<1$ is called the modulus of the Jacobi elliptic functions. When $k \rightarrow 0, \operatorname{sn}(\xi), \operatorname{cn}(\xi)$ and $\operatorname{dn}(\xi)$ degenerate to $\sin (\xi), \cos (\xi)$ and 1 respectively. While when $k \rightarrow 1, \operatorname{sn}(\xi), \operatorname{cn}(\xi)$ and $\operatorname{dn}(\xi)$ degenerate to $\tanh (\xi), \operatorname{sech}(\xi)$ and $\operatorname{sech}(\xi)$ respectively. 

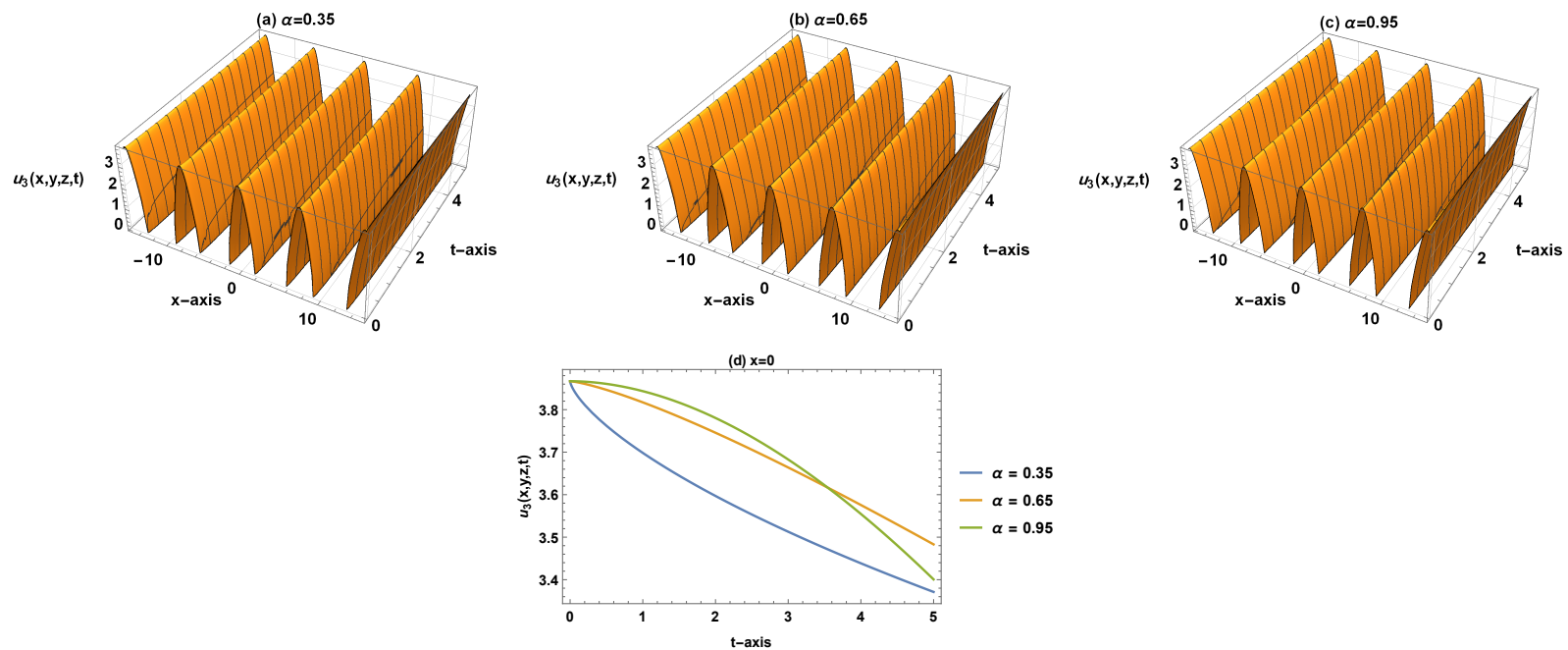

Fig. 3: (a)-(d) The wave solution $u_{3}(x, y, z, t)$ given by (24) in 3D- and 2D-plots when $y=z=0$.

Fig. 3 depicts the 3D and 2D charts of the solution given by $u_{3}(x, y, z, t)$ with the parameters $a=0.1, b=0.2$, and $k=0.5$.

\subsection{The rational solutions}

To find the rational solutions of the fractional $(3+1)$ Zakharov-Kuznetsov equation with power law nonlinearity, we assume that

$$
\begin{aligned}
& V(\xi)=\sum_{i=0}^{n} p_{i} \phi^{i}(\xi) / \sum_{i=0}^{r} q_{i} \phi^{i}(\xi), \quad n \geq r, \\
& \left(\phi^{\prime}(\xi)\right)^{p}=\sum_{i=0}^{p k} b_{i} \phi^{i}(\xi), \quad p=1,2,
\end{aligned}
$$

where $p_{i}, q_{i}$ and $b_{i}$ are arbitrary constants. By considering the homogeneous balance between $V V^{\prime \prime}$ and $V^{3}$ in Eq. (12), we get $n-r=2(k-1), k=1,2,3, \ldots$.

Here, we find these solutions when $k=1$ (so $n=r$ ) and $p=2$. So from (25), we have two cases as follow

\subsubsection{Case 1: periodic type}

In this case, we assume that

$$
\begin{gathered}
V(\xi)=\frac{p_{0}+p_{1} \phi(\xi)}{q_{0}+q_{1} \phi(\xi)}, \\
\phi^{\prime}(\xi)=\sqrt{b_{0}^{2}-b_{2}^{2} \phi^{2}(\xi)} .
\end{gathered}
$$

Similarly, when we use Eq. (26) in Eq. (12), we obtain a system of algebraic equations from the coefficients of poly- nomial of $\phi(\xi)$. By solving this algebraic system of equations, we get

$$
\begin{aligned}
& p_{0}=-\frac{9 b b_{0} b_{2} q_{1}}{2 a}, p_{1}=\frac{9 b b_{2}^{2} q_{1}}{2 a}, q_{0}=\frac{b_{0} q_{1}}{b_{2}}, \\
& \lambda=\frac{3 b b_{2}^{2}}{2}, n=2 .
\end{aligned}
$$

By solving the auxiliary equation $\phi^{\prime}(\xi)=$ $\sqrt{b_{0}^{2}-b_{2}^{2} \phi^{2}(\xi)}$ and substituting together with (27) into Eq. (26), we get the solution of eq. (1) namely

$$
u_{4}(x, y, z, t)=\left(\frac{9 b b_{2}^{2}\left(-1+\sin \left(b_{2} \xi\right)\right)}{2 a\left(1+\sin \left(b_{2} \xi\right)\right)}\right)^{\frac{1}{2}},
$$

where $\xi=x+y+z-\left(\frac{3 b b_{2}^{2}}{2 \alpha}\right) t^{\alpha}$ and $0<\alpha \leq 1$.

Fig. 4 depicts the $3 \mathrm{D}$ and 2D charts of the solution given by $u_{4}(x, y, z, t)$ with the parameters $a=0.1, b=$ -0.2 , and $b_{2}=1$.

\subsubsection{Case 2: soliton type}

Here, we assume that

$$
\begin{gathered}
V(\xi)=\frac{p_{0}+p_{1} \phi(\xi)}{q_{0}+q_{1} \phi(\xi)}, \\
\phi^{\prime}(\xi)=\sqrt{b_{0}+b_{1} \phi(\xi)+b_{2} \phi^{2}(\xi)} .
\end{gathered}
$$

When we use Eq. (29) in Eq. (12), we obtain a system of algebraic equations from the coefficients of polynomial of $\phi(\xi)$. By solving this algebraic system of equations, we get

$$
\begin{gathered}
p_{0}=-\frac{9 b q_{1}\left(b_{1}+H\right)}{4 a}, p_{1}=-\frac{9 b b_{2} q_{1}}{2 a}, \\
q_{0}=\frac{q_{1}\left(b_{1}-H\right)}{2 b_{2}}, \lambda=-\frac{3 b b_{2}}{2}, n=2,
\end{gathered}
$$



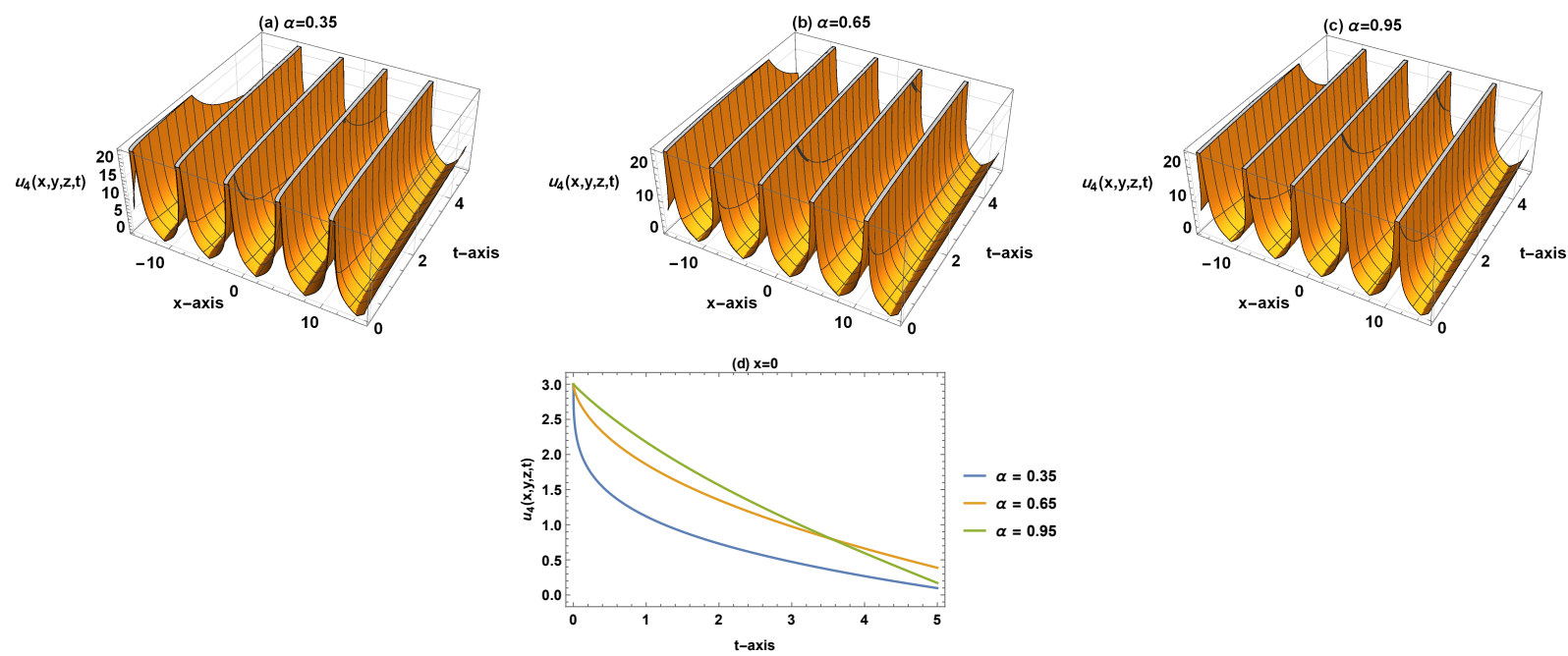

Fig. 4: (a)-(d) The wave solution $u_{4}(x, y, z, t)$ given by (28) in 3D- and 2D-plots when $y=z=0$.
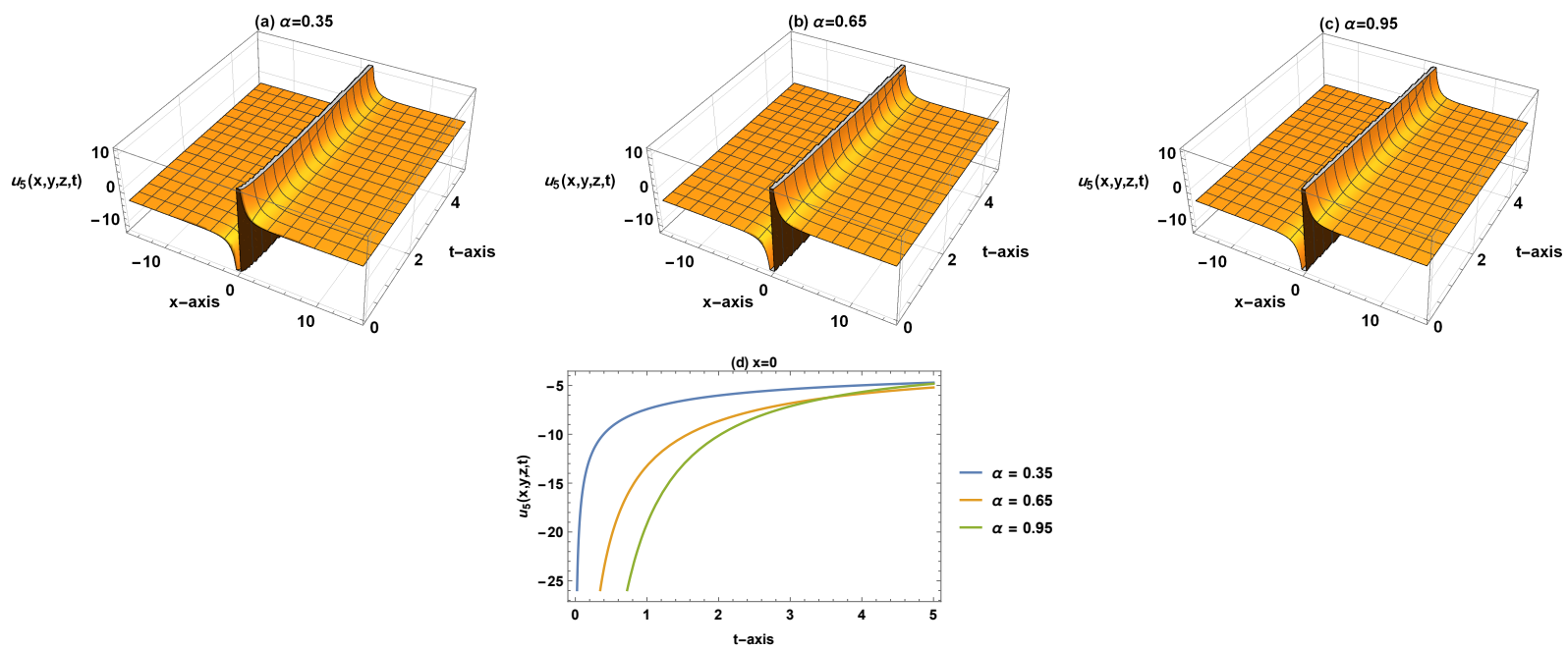

Fig. 5: (a)-(d) The wave solution $u_{5}(x, y, z, t)$ given by (31) in 3D- and 2D-plots when $y=z=0$.

where $H=\sqrt{b_{1}^{2}-4 b_{0} b_{2}}$.

By solving the auxiliary equation $\phi^{\prime}(\xi)=$ $\sqrt{b_{0}+b_{1} \phi(\xi)+b_{2} \phi^{2}(\xi)}$ and substituting together with (30) into Eq. (29), we get the solution of Eq. (1) namely

$$
u_{5}(x, y, z, t)=\left(\frac{3 \sqrt{-b b_{2}}\left(e^{\sqrt{b_{2}} \xi}+H\right)}{\sqrt{2 a}\left(e^{\sqrt{b_{2}} \xi}-H\right)}\right),
$$

where $\xi=x+y+z+\left(\frac{3 b b_{2}}{2 \alpha}\right) t^{\alpha}$ and $0<\alpha \leq 1$.

Fig. 5 depicts the 3D and 2D charts of the solution given by $u_{5}(x, y, z, t)$ with the parameters $a=0.1, b=$ $-0.2, H=1$, and $b_{2}=1$.

\section{Conclusion}

In this work, we have constructed exact traveling wave solutions for nonlinear conformable fractional evolution equations by using unified method. This method allows us to solve more nonlinear conformable fractional evolution equations in mathematical physics via the $(3+1)$ dimensional conformable fractional Zakharov-Kuzetsov equation with power law nonlinearity. As a result, many new types of exact traveling wave solutions are obtained. These solutions include the solitary wave solutions, soliton wave solutions, elliptic wave solutions, and periodic (hyperbolic) wave rational solutions. 


\section{References}

[1] Iyiola, O.S., Tasbozan, O., Kurt, A., Çenesiz, Y., (2017). On the analytical solutions of the system of conformable timefractional Robertson equations with 1-D diffusion. Chaos, Solitons \& Fractals, 94, 1-7

[2] Kurt, A., Tasbozan, O., Baleanu, D., (2017). New solutions for conformable fractional Nizhnik-Novikov-Veselov system via G'/G expansion method and homotopy analysis methods. Optical and Quantum Electronics, 49(10), 333

[3] Tasbozan, O., Çenesiz, Y., Kurt, A., Baleanu, D., (2017). New analytical solutions for conformable fractional PDEs arising in mathematical physics by exp-function method. Open Physics, 15(1), 647-651

[4] Tasbozan, O., Şenol, M., Kurt, A, Özkan, O., (2018). New solutions of fractional Drinfeld-Sokolov-Wilson system in shallow water waves. Ocean Engineering, 161, 62-68

[5] Çenesiz, Y., Kurt, A., Tasbozan, O., (2017). On the New Solutions of the Conformable Time Fractional Generalized HirotaSatsuma Coupled KdV System. Annals of West University of Timisoara-Mathematics and Computer Science, 55(1), 37-50

[6] Orkun Tasbozan, Yucel Çenesiz, Ali Kurt, Olaniyi S. Iyiola, (2018). New Analytical Solutions and Approximate Solution of the Space-Time Conformable Sharma-Tasso-Olver Equation. Progress in Fractional Differentiation and Applications, 4(4), 519-531

[7] Kurt, A., Rezazadeh, H., Senol, M., Neirameh, A., Tasbozan, O., Eslami, M., Mirzazadeh, M., (2018). Two effective approaches for solving fractional generalized HirotaSatsuma coupled KdV system arising in interaction of long waves. Journal of Ocean Engineering and Science https://doi.org/10.1016/j.joes.2018.12.004

[8] Kurt, A., Tasbozan, O., (2018, December). Approximate Analytical Solutions to Conformable Modified Burgers Equation Using Homotopy Analysis Method. Annales Mathematicae Silesianae. Sciendo

[9] Orkun TAŞBOZAN, Ali KURT, (2018). New Travelling Wave Solutions for Time-Space Fractional Liouville and Sine-Gordon Equations. Journal of the Institute of Science and Technology, 8(4), 295-303

[10] Podlubny, I., (1998). Fractional differential equations, (Vol. 198). Academic press

[11] Miller, K. S., Ross, B., (1993). An introduction to the fractional calculus and fractional differential equations

[12] Khalil, R., Al Horani, M., Yousef, A., Sababheh, M., (2014). A new definition of fractional derivative. Journal of Computational and Applied Mathematics, 264, 65-70

[13] Abdeljawad, T., (2015). On conformable fractional calculus. Journal of computational and Applied Mathematics, 279, 57-66

[14] Chung, W. S., (2015). Fractional Newton mechanics with conformable fractional derivative. Journal of Computational and Applied Mathematics, 290, 150-158

[15] Rezazadeh, H., Aminikhah, H., Refahi Sheikhani, A. H., (2017). Stability Analysis of Conformable Fractional Systems. Iranian Journal of Numerical Analysis and Optimization, 7(1), 13-32

[16] Zheng, A., Feng, Y., Wang, W., (2015). The Hyers-Ulam stability of the conformable fractional differential equation. Mathematica Aeterna, 5(3), 485-492
[17] Kurt, A., Cenesiz, Y., Tasbozan, O., (2015). On the solution of Burgers' equation with the new fractional derivative. Open Phys, 13(1), 355-360

[18] Zhao, D., Luo, M., (2017). General conformable fractional derivative and its physical interpretation. Calcolo, 54(3), 903917

[19] Eslami, M., Rezazadeh, H., (2016). The first integral method for Wu-Zhang system with conformable time-fractional derivative. Calcolo, 53(3), 475-485

[20] Eslami, M., (2016). Exact traveling wave solutions to the fractional coupled nonlinear Schrodinger equations. Applied Mathematics and Computation, 285, 141-148

[21] Kaplan, M., (2017). Applications of two reliable methods for solving a nonlinear conformable time-fractional equation. Optical and Quantum Electronics, 49(9), 312

[22] Korkmaz, A., (2017). Exact Solutions to (3+1) Conformable Time Fractional Jimbo-Miwa, Zakharov-Kuznetsov and Modified Zakharov-Kuznetsov Equations. Communications in Theoretical Physics, 67(5), 479

[23] Aminikhah, H., Sheikhani, A. R., Rezazadeh, H., (2016). Subequation method for the fractional regularized long-wave equations with conformable fractional derivatives. Scientia Iranica. Transaction B, Mechanical Engineering, 23(3), 1048

[24] Rezazadeh, H., Ziabarya, B. P., (2016). Sub-equation method for the conformable fractional generalized kuramoto sivashinsky equation. Computational Research Progress in Applied Science Engineering, 2(3), 106-109

[25] Khodadad, F. S., Nazari, F., Eslami, M., Rezazadeh, H., (2017). Soliton solutions of the conformable fractional ZakharovKuznetsov equation with dual-power law nonlinearity. Optical and Quantum Electronics, 49(11), 384

[26] Tariq, H., Akram, G., (2017). New traveling wave exact and approximate solutions for the nonlinear Cahn-Allen equation: evolution of a nonconserved quantity. Nonlinear Dynamics, 88(1), 581-594

[27] Akbulut, A., Kaplan, M., (2018). Auxiliary equation method for time-fractional differential equations with conformable derivative. Computers \& Mathematics with Applications, 75(3), 876-882

[28] Ekici, M., Mirzazadeh, M., Eslami, M., Zhou, Q., Moshokoa, S. P., Biswas, A., Belic, M., (2016). Optical soliton perturbation with fractional-temporal evolution by first integral method with conformable fractional derivatives. Optik-International Journal for Light and Electron Optics, 127(22), 10659-10669

[29] Çenesiz, Y., Baleanu, D., Kurt, A., Tasbozan, O., (2017). New exact solutions of Burgers' type equations with conformable derivative. Waves in Random and Complex Media, 27(1), 103116

[30] Kurt, A., Tasbozan, O., Çenesiz, Y., Baleanu, D., (2016). New Exact Solutions for Some Nonlinear Conformable PDEs Using Exp-Function Method. In International Conference on Applied Mathematics and Analysis in Memory of Gusein Sh. Guseinov. Atilim University Ankara, Turkey.

[31] Tasbozan, O., Çenesiz, Y., Kurt, A., (2016). New solutions for conformable fractional Boussinesq and combined KdV-mKdV equations using Jacobi elliptic function expansion method. The European Physical Journal Plus, 131(7), 244

[32] Eslami, M., Rezazadeh, H., Rezazadeh, M., Mosavi, S. S., (2017). Exact solutions to the space-time fractional Schrödinger-Hirota equation and the space-time modified 
KDV-Zakharov-Kuznetsov equation. Optical and Quantum Electronics, 49(8), 279

[33] Eslami, M., Khodadad, F. S., Nazari, F., Rezazadeh, H., (2017). The first integral method applied to the Bogoyavlenskii equations by means of conformable fractional derivative. Optical and Quantum Electronics, 49(12), 391

[34] Hosseini, K., Mayeli, P., Ansari, R., (2018). Bright and singular soliton solutions of the conformable time-fractional KleinGordon equations with different nonlinearities. Waves in Random and Complex Media, 28(3), 426-434

[35] Eslami, M., (2017). Solutions for Space Time Fractional (2+1)Dimensional Dispersive Long Wave Equations. Iranian Journal of Science and Technology, Transactions A: Science, 41(4), 1027-1032

[36] Cenesiz, Y., Tasbozan, O., Kurt, A., (2017). Functional Variable Method for conformable fractional modified KdV-ZK equation and Maccari system. Tbilisi Mathematical Journal, 10(1), 118126

[37] Kaplan, M., Bekir, A., Ozer, M. N., (2017). A simple technique for constructing exact solutions to nonlinear differential equations with conformable fractional derivative. Optical and Quantum Electronics, 49(8), 266

[38] Wazwaz, A. M., (2005). Exact solutions with solitons and periodic structures for the Zakharov-Kuznetsov (ZK) equation and its modified form. Communications in Nonlinear Science and Numerical Simulation, 10(6), 597-606

[39] Abdel Gawad, H. I., Tantawy, M., Osman, M. S., (2016). Dynamic of DNA's possible impact on its damage. Mathematical Methods in the Applied Sciences, 39(2), 168-176

[40] Abdel-Gawad, H. I., Osman, M., (2015). On shallow water waves in a medium with time-dependent dispersion and nonlinearity coefficients. Journal of advanced research, 6(4), 593 599

[41] Osman, M. S., Machado, J. A. T., Baleanu, D., (2018). On nonautonomous complex wave solutions described by the coupled Schrödinger-Boussinesq equation with variable-coefficients. Optical and Quantum Electronics, 50(2), 73

[42] Osman, M. S., Korkmaz, A., Rezazadeh, H., Mirzazadeh, M., Eslami, M., Zhou, Q., (2018). The Unified Method for Conformable Time Fractional Schrödinger Equation with Perturbation Terms. Chinese Journal of Physics, 56(5), 2500-2506

[43] Osman, M. S., Abdel-Gawad, H. I., El Mahdy, M. A., (2018). Two-layer-atmospheric blocking in a medium with high nonlinearity and lateral dispersion. Results in physics, 8, 1054-1060
[44] Abdel-Gawad, H. I., Osman, M., (2014). Exact solutions of the Korteweg-de Vries equation with space and time dependent coefficients by the extended unified method. Indian Journal of Pure and Applied Mathematics, 45(1), 1-12

[45] Abdel-Gawad, H. I., Osman, M. S., (2013). On the variational approach for analyzing the stability of solutions of evolution equations. Kyungpook mathematical journal, 53(4), 661-680

[46] Osman, M. S., (2017). Nonlinear interaction of solitary waves described by multi-rational wave solutions of the $(2+1)$ dimensional Kadomtsev Petviashvili equation with variable coefficients. Nonlinear Dynamics, 87(2), 1209-1216

[47] Osman, M. S., Abdel-Gawad, H. I., (2015). Multi-wave solutions of the (2+1)-dimensional Nizhnik-Novikov-Veselov equations with variable coefficients. The European Physical Journal Plus, 130(10), 215

[48] Osman, M. S., (2017). Analytical study of rational and doublesoliton rational solutions governed by the KdV SawadaKotera Ramani equation with variable coefficients. Nonlinear Dynamics, 83(3), 2283-2289

[49] Osman, M. S., Machado, J. A. T., (2018). New nonautonomous combined multi-wave solutions for (2+1)-dimensional variable coefficients KdV equation. Nonlinear Dynamics, 93(2), 733-740

[50] Wazwaz, A. M., Osman, M. S., (2018). Analyzing the combined multi-waves polynomial solutions in a two-layer-liquid medium. Computers \& Mathematics with Applications, 76(2), 276-283

[51] Osman, M. S., Machado, J. A. T., (2018). The dynamical behavior of mixed-type soliton solutions described by $(2+1)$ dimensional Bogoyavlensky-Konopelchenko equation with variable coefficients. Journal of Electromagnetic Waves and Applications, 32(11), 1457-1464

[52] Matebese, B. T., Adem, A. R., Khalique, C. M., Biswas, A., (2011). Solutions of Zakharov-Kuznetsov equation with power law nonlinearity in $(1+3)$ dimensions. Physics of Wave Phenomena, 19(2), 148-154

[53] Zhong-Zhou, D., Yong, C., Yan-Huai, L., (2010). Symmetry reduction and exact solutions of the $(3+1)$-dimensional Zakharov-Kuznetsov equation. Chinese Physics B, 19(9), 090205

[54] Aminikhah, H., Pourreza Ziabary, B., Rezazadeh, H., (2015). Exact traveling wave solutions of partial differential equations with power law nonlinearity. Nonlinear Engineering, 4(3), 181188

[55] Zhang, L. H., (2009). Travelling wave solutions for the generalized Zakharov-Kuznetsov equation with higher-order nonlinear terms. Applied Mathematics and Computation, 208(1), 144-155 